\title{
Plant sterol-enriched milk tea decreases blood cholesterol concentrations in Chinese adults: a randomised controlled trial
}

\author{
Nicole Y. $\mathrm{Li}^{1}{ }^{*}, \mathrm{Keji}_{\mathrm{Li}^{2}}$, Zhi Qi ${ }^{2}$, Isabelle Demonty ${ }^{3}$, Michelle Gordon ${ }^{3}$, Lesley Francis ${ }^{1}$, \\ Henri O. F. Molhuizen ${ }^{3}$ and Bruce C. Neal ${ }^{1}$ \\ ${ }^{1}$ The George Institute for International Health, University of Sydney, PO Box M201, Camperdown, Sydney, NSW2050, Australia \\ ${ }^{2}$ School of Public Health, Peking University Health Science Centre, Peking, China \\ ${ }^{3}$ Unilever Food and Health Research Institute, Rotterdam, The Netherlands \\ (Received 19 December 2006 - Revised 30 March 2007 - Accepted 12 April 2007)
}

\begin{abstract}
The cholesterol-lowering effects of plant sterols in a format suitable for use in China have not previously been investigated. We conducted the study to quantify in adult Chinese the effects on blood lipid concentrations of a plant sterol-enriched milk tea powder. The study was a double-blind, randomised trial in which 309 participants were randomised to receive daily 2.3 or $1.5 \mathrm{~g}$ plant sterol supplementation or placebo for 5 weeks. The milk tea was consumed with the two fattiest meals of the day with half the assigned daily dose taken on each occasion. Fasting venous blood samples were collected before commencement and upon completion of randomised treatment. The mean age of study participants was 44 years, $62 \%$ were female and $62 \%$ had a history of hypercholesterolaemia. Baseline mean total cholesterol was $5.5 \mathrm{mmol} / 1$ and LDL-cholesterol was $3.2 \mathrm{mmol} / \mathrm{l}$. Compared with placebo, the $2.3 \mathrm{~g} / \mathrm{d}$ plant sterol dose reduced total cholesterol by $0.25(95 \% \mathrm{CI} 0.07,0.43) \mathrm{mmol} / \mathrm{l}(P=0.01)$ and the $1.5 \mathrm{~g} / \mathrm{d}$ dose by $0.23(95 \% \mathrm{CI} 0.06,0.41) \mathrm{mmol} / \mathrm{l}(P=0.01)$. For LDL-cholesterol the corresponding reductions were $0.17(95 \% \mathrm{CI} 0.00$, $0.35) \mathrm{mmol} / \mathrm{l}(P=0.06)$ and $0.15(95 \% \mathrm{CI}-0.02,0.32) \mathrm{mmol} / \mathrm{l}(P=0.08)$. For neither outcome was there evidence of differences between the effects of the two doses (both $P$ values $>0.4$ ). In conclusion, the consumption of plant sterol-enriched milk tea decreased cholesterol concentrations although to a lesser extent than was anticipated. The reason for reduced efficacy is unclear but may be attributable to the novel food format used or the Chinese population studied.
\end{abstract}

Plant sterols: China: Randomised controlled trials: Cholesterol

It is well established that higher concentrations of blood total cholesterol and blood LDL-cholesterol are associated with higher rates of ischaemic $\mathrm{CVD}^{1,2}$. Plant sterols are naturally occurring substances ${ }^{3}$ that inhibit the intestinal absorption of cholesterol $^{4,5}$ and reduce total and LDL-cholesterol concentrations when administered as dietary supplements 5 . They have effects in a broad range of individuals ${ }^{6,7}$ and are active both alone and when used with drug-based cholesterol lowering $^{6,8}$. Products enriched with plant sterols are registered as functional foods in many countries 5 on the basis of their effects on blood cholesterol concentrations and presumed consequent protection against ischaemic cardiovascular events.

Dietary fat consumption in China has soared from $58 \mathrm{~g} /$ individual per d in 1992 to 76 g/individual per d in 2002 with a corresponding negative impact on blood cholesterol concentrations ${ }^{9}$ and the burden of $\mathrm{CVD}^{10}$. Plant sterolenriched foods might have a role to play in the control of CVD in China but evidence of their effectiveness in the population is required before their introduction. As such, the primary aim of the China Plant Sterol Trial was to precisely and reliably define the effects on blood lipid concentrations of plant sterols compared with placebo in healthy Chinese adults. A novel powdered milk tea format was developed to suit the cultural setting and two different doses were studied because the dose of plant sterol appropriate for this food format has not been established.

\section{Participants and methods}

The China Plant Sterol Trial was a double-blind, randomised controlled trial done between April and June 2006. The trial was approved by the Ethics Committees of the University of Sydney, Australia, the Peking University, China and Unilever Foods in the Netherlands. All participants provided written informed consent and the study was conducted according to the principles of the Declaration of Helsinki and subsequent amendments.

\section{Participants}

Participants were recruited from three community-based clinical research centres in Beijing, China. Individuals were potentially eligible if they were (a) aged 18-65 years old; (b) able to provide informed consent; (c) considered by the centre 
physician as likely to adhere to the study treatment and follow-up schedule. Participants were also required to (d) consume a usual diet containing a significant amount of fat and/or have a history of hypercholesterolaemia, in an effort to achieve in the study participants a higher average baseline cholesterol level than that of the general Chinese population. A usual diet containing a significant amount of fat was defined as one in which twelve or more meals per week were reported to include animal fat, deep-fried food, eggs, offal or full-fat dairy products. A history of hypercholesterolaemia was based on a public hospital laboratory print-out dated within the past 2 years and showing a fasting total cholesterol concentration of $5.7 \mathrm{mmol} / \mathrm{l}(2200 \mathrm{mg} / \mathrm{l})$ or above. Individuals using statins were eligible.

Participants were excluded if there was a known contraindication to plant sterol use, such as a prior adverse reaction to plant sterol-enriched foods or a history of sitosterolaemia. Individuals were also excluded if efficacy of plant sterol treatment was likely to be reduced through serious gastrointestinal disease or use of a drug with a significant impact on gastrointestinal absorption of cholesterol. Other exclusions were on the basis of serious illness, lactose intolerance or requirement for a diet that excluded dairy products, current hospitalisation, current use of plant sterols, participation in another study within the previous 3 months or pregnant or planning to become pregnant within the following 3 months.

\section{Run-in, randomisation and follow-up}

All individuals interested in participating in the trial attended a baseline visit to assess eligibility. Potentially eligible participants commenced a placebo run-in period of between 10 to $20 \mathrm{~d}$ duration to confirm their suitability for enrolment. Participants that returned to the study clinic on the day scheduled for the randomisation visit, who reported having taken at least $95 \%$ of the scheduled run-in treatment and were willing to continue were subsequently randomised. Randomisation was done in real time using an independent computerised randomisation service accessed via the Internet and maintained by the George Institute in Australia. The randomisation service provided a unique number corresponding to a treatment pack held at the study research centre. Each treatment pack contained all of the post-randomisation study treatment needed for that individual. A minimisation algorithm incorporating current use of statin therapy, history of hypercholesterolaemia and clinical centre was used to ensure balance across the three study groups in these key participant characteristics. Randomised participants were then seen at the follow-up visit which was scheduled for 30-40 d after randomisation. Study data were collected in Beijing and entered through a secure study website, with data management and primary analyses done at The George Institute (Sydney, Australia).

\section{Study treatment}

Run-in treatment comprised placebo milk tea taken twice daily for 10 to $20 \mathrm{~d}$ and post-randomisation treatment was milk tea containing either 2.3 or $1.5 \mathrm{~g}$ plant sterols/d or placebo taken for $30-40 \mathrm{~d}$. The milk tea preparation contained black tea powder; for the formulation, see Table 1. Both run-in and post-randomisation treatment were consumed with the two
Table 1. Macronutrient composition of the milk tea products used in the trial $^{*}$

\begin{tabular}{lccc}
\hline $\begin{array}{l}\text { Per daily portion } \\
\text { of } 30 \mathrm{~g}\end{array}$ & $\begin{array}{c}\text { Control milk } \\
\text { tea powder }\end{array}$ & $\begin{array}{c}\text { Milk tea powder } \\
\text { providing } 1.5 \mathrm{~g} \\
\text { plant sterols/d }\end{array}$ & $\begin{array}{c}\text { Milk tea powder } \\
\text { providing 2.3 g } \\
\text { plant sterols/d }\end{array}$ \\
\hline $\begin{array}{l}\text { Energy (kJ)* } \\
\text { Proteins (g) }\end{array}$ & 442 & 531 & 487 \\
Carbohydrates (g) & $1 \cdot 7$ & $1 \cdot 1$ & 1.4 \\
Fat (g) & $25 \cdot 2$ & 21.4 & $20 \cdot 3$ \\
PUFA (\%) & $2 \cdot 3$ & $4 \cdot 7$ & $5 \cdot 2$ \\
MUFA (\%) & 62 & 48 & 48 \\
SFA (\%) & 30 & 28 & 28 \\
Plant sterols $(\mathrm{g})$ & 8 & 24 & 24 \\
\hline
\end{tabular}

*Calculated values from the recipe; otherwise, measured values.

fattiest meals of the day with half the assigned daily treatment taken on each occasion. A rigorous quality-control procedure including full biochemical analysis of thirty randomly selected treatment packs showed complete accuracy of sample packaging and plant sterol levels.

To maximise adherence and simplify the treatment regimen, each daily dose of milk tea was provided in two pre-packaged sachets, the contents of which were dissolved in boiled water $\left(80-90^{\circ} \mathrm{C}\right)$ immediately before consumption. Participants were encouraged to continue with their normal diet and exercise patterns during the study period, and for those using other cholesterol-lowering medications the usual dose of treatment was continued throughout the study. Dietary records were not kept. Treatment allocation was concealed from investigators and participants until all follow up was complete and the database was locked.

\section{Outcomes}

The primary outcome was LDL-cholesterol. Secondary outcomes were total cholesterol, HDL-cholesterol and TAG. Blood samples were collected at the randomisation visit and at the follow-up visit with participants fasted overnight on both occasions. Lipids were measured directly using a Hitachi 7170 Auto-Analyser located at the Central Laboratory of Peking University Third Hospital, a system approved by the Cholesterol Reference Method Laboratory Network of the Centers for Disease Control and Prevention. The tertiary outcomes were liking of study treatment evaluated by nine different measures (overall taste of the milk tea; the milk taste; the tea taste; the balance of the milk and tea taste; the strength of sweetness; the feel in the mouth; the aftertaste; the strength of smoothness; the darkness of the colour) recorded on visual analogue scales and adherence (defined as the number of tea sachets used divided by the number of tea sachets due to have been used). Participants were asked to bring unused tea sachets to the study clinic at the follow-up visit for counting.

\section{Statistics}

Power. The study was designed to provide $80 \%$ power $(\alpha=0.05)$ to detect a $10 \%$ or greater difference in LDLcholesterol concentrations between any pair of treatment groups. There was similar power to detect an $8.5 \%$ or greater difference between the two active treatment groups combined 
( $n$ 200) and placebo ( $n$ 100). These power calculations were based upon data about population cholesterol concentrations in urban Chinese individuals collected by previous studies $^{10,11}$.

Analysis. Analysis of treatment effects was by intention to treat using procedures available in the SAS System for Windows version 8 (SAS Institute Inc., Cary, NC, USA). The baseline to follow-up changes in study outcomes were calculated for each randomised group and compared between them using ANOVA with $C I$ and $P$ values calculated for the differences $^{12}$. Pre-defined subgroup analyses exploring the constancy of the effects of the randomised treatment on LDL-cholesterol and total cholesterol were done by including an interaction term in the relevant models. For continuous baseline measures median values were used to split participants into subgroups except for total cholesterol where Chinese regulations concerning the registration of functional foods recommend a cut-off point of $5.2 \mathrm{mmol} / \mathrm{l}$. The subgroup analyses were focused on the comparison of effects between the two active treatment groups combined $v$. placebo in an effort to maximise reliability of the findings. The data describing the percentage differences in lipid concentrations and the adherence to study treatment were tested using the non-parametric Kruskal-Wallis test since the data were skewed ${ }^{12}$. While the data about liking of study treatment were also skewed they were successfully transformed using an Arcsin (square root) transformation and were tested using ANOVA.

\section{Results}

Registration, randomisation, adherence and follow up

A total of 432 individuals were registered and 309 were randomised (Fig. 1). Failure of registered participants to proceed to randomisation was primarily a consequence of inadequate

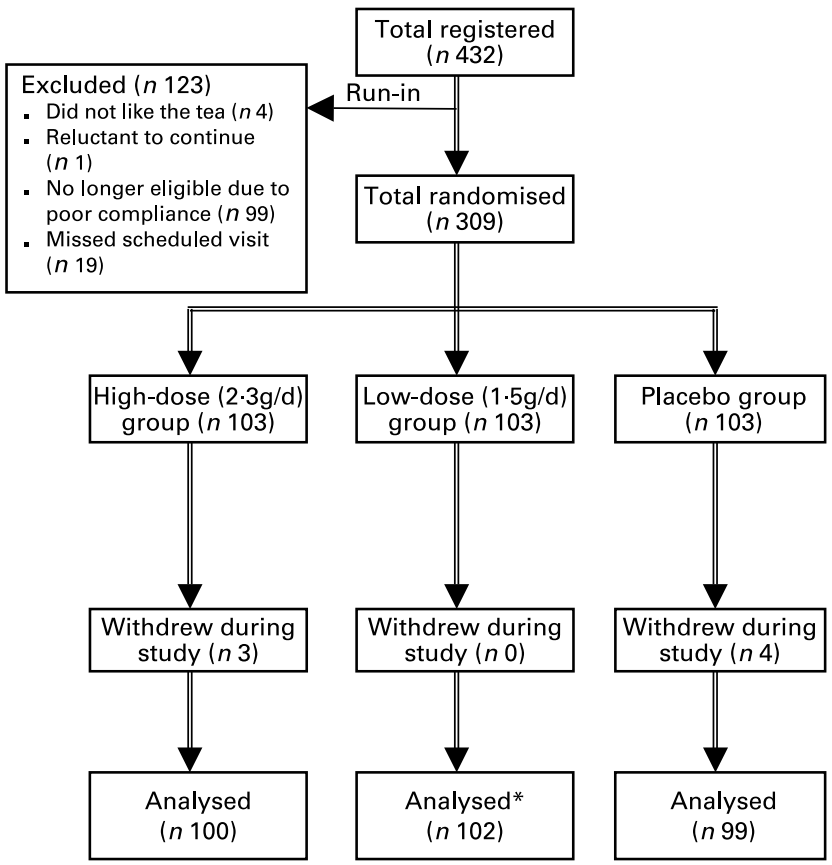

Fig. 1. Study flow chart. *Analysis of one blood sample was not possible due to extensive haemolysis. adherence to study treatment during the run-in phase. Mean adherence after randomisation was $97 \%$ in both active treatment groups and $95 \%$ in the placebo group, with no difference in adherence detected between any pair of randomised groups (all $P>0.25$ ). The final follow-up visit was attended by 302 $(98 \%)$ randomised participants, and baseline and follow-up blood samples from 301 (97\%) were available for analysis.

\section{Baseline characteristics}

Randomised participants had a mean age of 44 years and $62 \%$ were female (Table 2). Almost two-thirds had a history of hypercholesterolaemia but few were using statins or any other type of cholesterol-lowering agent. Mean BMI was $26 \mathrm{~kg} / \mathrm{m}^{2}$, total cholesterol $5.5 \mathrm{mmol} / \mathrm{l}$, LDL-cholesterol $3.2 \mathrm{mmol} / \mathrm{l}$, HDL-cholesterol $1.5 \mathrm{mmol} / \mathrm{l}$ and TAG $2.1 \mathrm{mmol} / \mathrm{l}$.

\section{Effects of randomised treatment on blood lipids}

Compared with placebo, both doses of plant sterols brought about significant absolute and percentage reductions in total cholesterol, with no effect on either HDL-cholesterol or TAG (Fig. 2 and Table 3). The same was also true for each of these three lipid outcomes for the analyses which treated the two doses of active treatment as one and compared them against placebo. For the primary outcome of LDL-cholesterol there was a significant percentage reduction achieved with the higher dose of plant sterol but not the lower dose and for neither dose did the absolute reductions in LDL-cholesterol reach conventional levels of statistical significance. There were, however, significant absolute and proportional reductions in LDL-cholesterol for the analyses which treated the two doses of active treatment as one and compared them against placebo. For none of the four lipid parameters did the head-to-head comparisons between doses show evidence of a difference in effects between the 2.3 and $1.5 \mathrm{~g}$ doses of plant sterols.

The subgroup analyses identified no differences in the effects of randomised treatment on LDL-cholesterol or total cholesterol in the pre-specified participant subgroups defined by baseline total cholesterol concentration, sex, age, baseline LDL-cholesterol concentration or reported level of fat consumption (all $P$ interaction $>0.08$ ). Nor was there any difference in the effects of randomised treatment on these outcomes between subgroups defined post hoc on the basis of BMI (all $P>0 \cdot 5)$.

\section{Effects of randomised treatment on liking of milk tea and other outcomes}

For none of the comparisons between randomised groups were there any differences detected in the nine aspects of liking recorded (all $P>0 \cdot 10$ ). Nor were there any serious adverse drug reactions or serious adverse events documented.

\section{Discussion}

The present study showed that twice-daily dietary supplementation with plant sterol-enriched milk tea at a dose of either 1.5 or $2.3 \mathrm{~g} / \mathrm{d}$ reduced blood total cholesterol concentrations in healthy urban Chinese. Corresponding clear effects on 
Table 2. Baseline characteristics of study participants by randomised group (Mean values and standard deviations or proportions)

\begin{tabular}{|c|c|c|c|c|c|c|}
\hline \multirow[b]{2}{*}{ Group... } & \multicolumn{2}{|c|}{$2.3 \mathrm{~g}$ plant sterols $/ \mathrm{d}$} & \multicolumn{2}{|c|}{$1.5 \mathrm{~g}$ plant sterols $/ \mathrm{d}$} & \multicolumn{2}{|c|}{ Placebo } \\
\hline & Mean & SD & Mean & SD & Mean & SD \\
\hline Female (\%) & \multicolumn{2}{|c|}{57} & \multicolumn{2}{|c|}{61} & \multicolumn{2}{|c|}{69} \\
\hline Age (years) & 44 & $12 \cdot 3$ & 44 & $12 \cdot 2$ & 45 & 11.0 \\
\hline \multicolumn{7}{|l|}{ Disease history (\%) } \\
\hline Stroke or transient ischaemic attack & \multicolumn{2}{|c|}{1} & \multicolumn{2}{|c|}{4} & \multicolumn{2}{|c|}{1} \\
\hline Myocardial infarction or unstable angina & \multicolumn{2}{|c|}{4} & \multicolumn{2}{|c|}{0} & \multicolumn{2}{|c|}{0} \\
\hline Congestive heart failure & \multicolumn{2}{|c|}{0} & \multicolumn{2}{|c|}{0} & \multicolumn{2}{|c|}{0} \\
\hline Hypertension & \multicolumn{2}{|c|}{22} & \multicolumn{2}{|c|}{22} & \multicolumn{2}{|c|}{24} \\
\hline Hypercholesterolaemia & \multicolumn{2}{|c|}{63} & \multicolumn{2}{|c|}{61} & \multicolumn{2}{|c|}{61} \\
\hline Diabetes & \multicolumn{2}{|c|}{2} & \multicolumn{2}{|c|}{3} & \multicolumn{2}{|c|}{3} \\
\hline \multicolumn{7}{|l|}{ Medication usage (\%) } \\
\hline Statin & \multicolumn{2}{|c|}{2} & \multicolumn{2}{|c|}{1} & \multicolumn{2}{|c|}{0} \\
\hline Other cholesterol-lowering drug & \multicolumn{2}{|c|}{6} & \multicolumn{2}{|c|}{6} & & \\
\hline Any blood pressure-lowering drug & & & & & & \\
\hline Diet and other behaviours & & & & & & \\
\hline Number of meals high in fat eaten each week & & & & & & \\
\hline Current cigarette smoker (\%) & & & & & & \\
\hline Drinks alcohol (\%) & & & & & & \\
\hline Physical characteristics & & & & & & \\
\hline BMI $\left(\mathrm{kg} / \mathrm{m}^{2}\right)$ & 26 & 3.8 & 26 & 3.9 & 26 & $3 \cdot 6$ \\
\hline Waist circumference (cm) & 86 & $10 \cdot 8$ & 84 & $10 \cdot 8$ & 84 & $10 \cdot 9$ \\
\hline Blood pressure $(\mathrm{mmHg})$ & $117 / 77$ & $13 / 10$ & $117 / 77$ & $13 / 10$ & $118 / 77$ & $13 / 9$ \\
\hline Blood lipid concentrations $(\mathrm{mmol} / \mathrm{l})$ & & & & & & \\
\hline Total cholesterol & $5 \cdot 3$ & 1.2 & $5 \cdot 6$ & 1.3 & $5 \cdot 5$ & $1 \cdot 1$ \\
\hline LDL-cholesterol & $3 \cdot 1$ & 1.0 & $3 \cdot 2$ & 1.1 & 3.3 & 0.9 \\
\hline HDL-cholesterol & 1.4 & 0.4 & 1.4 & 0.3 & 1.5 & 0.4 \\
\hline TAG & $2 \cdot 0$ & 1.4 & $2 \cdot 3$ & $2 \cdot 0$ & 1.9 & 1.3 \\
\hline
\end{tabular}

LDL-cholesterol were not apparent for either dose although there were trends towards reduction for both and analyses done combining the two active doses to maximise statistical power did show significant falls in LDL-cholesterol. However, for both total cholesterol and LDL-cholesterol the effects of the plant sterols were less than anticipated on the basis of previous studies done mostly in Western populations and with other food formats 5 . While even the moderate lipid changes achieved in the study would be expected to translate into reduced risks of ischaemic $\mathrm{CVD}^{1,2}$, large numbers of individuals would require long-term treatment with this product for significant health benefits to accrue in the Chinese population.

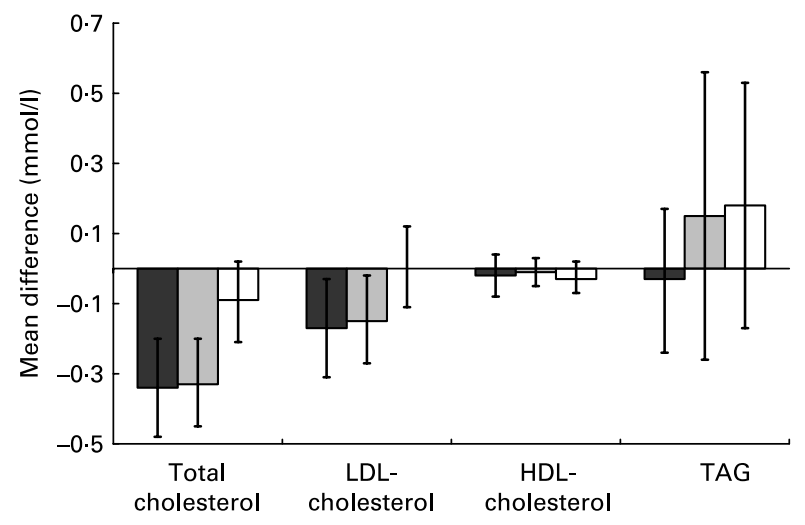

Fig. 2. Differences in blood lipid concentrations between baseline and follow up (mmol/l) by randomised group: ( $\square$ ), $2.3 \mathrm{~g}$ plant sterols $/ \mathrm{d}$; $(\square), 1.5 \mathrm{~g}$ plant sterols/d; $\square$, placebo. Values are mean point estimates of the observed effect, with vertical lines representing $95 \% \mathrm{Cl}$.
In a recent meta-analysis of randomised trials an average $2 \mathrm{~g} / \mathrm{d}$ dose of plant sterols reduced blood concentrations of LDL-cholesterol by about 0.43 (95\% CI $0.37,0.47) \mathrm{mmol} / \mathrm{l}$ amongst participants aged 40-49 years, with greater effects among older individuals and lesser effects among younger individuals 5 . In the present study of individuals with a mean age of 44 years the reduction in blood LDL-cholesterol concentration was only about one-third of that $(0.16(95 \% \mathrm{CI}$ $0.01,0.32) \mathrm{mmol} / \mathrm{l}$ ). The difference between the present study and the meta-analysis of prior studies appears unlikely to have arisen by chance. Neither does it appear to reflect differences in baseline cholesterol levels which were only moderately different between the present study and the overview (median $3.8 \mathrm{mmol} / \mathrm{l}$ in the meta-analysis $v$. mean $3.2 \mathrm{mmol} / \mathrm{l}$ in the present study), a difference insufficient to explain the approximate threefold difference in the proportionate lipid reductions observed $(9 \%$ in the meta-analysis and $3 \%$ in the present study).

The reason why the plant sterol produced only limited reductions in total and LDL-cholesterol in the present study cannot be stated with certainty. Ethno-specific lesser effects of cholesterol-lowering interventions are not supported by prior studies ${ }^{7,13-15}$ and lower levels of fat in the diets of the trial participants also appears an unlikely explanation ${ }^{16-19}$. Consumption of the milk tea outside of meal times may have occurred but it would not be expected to reduce efficacy to this extent ${ }^{4,19}$. It is possible that beverage formats may be a less effective delivery mechanism for plant sterols than other food formats with one prior study of a beverage format showing a significant fall in blood lipid levels ${ }^{20}$ but another showing no effect ${ }^{21}$. Inadequate dosing of plant sterol also seems an 
Table 3. Absolute and proportional reductions in blood lipids achieved with randomised treatment

\begin{tabular}{|c|c|c|c|c|c|c|c|c|}
\hline & \multicolumn{2}{|c|}{$2.3 \mathrm{~g} / \mathrm{d} v$. placebo } & \multicolumn{2}{|c|}{$1.5 \mathrm{~g} / \mathrm{d} v$. placebo } & \multicolumn{2}{|c|}{$1.5 \mathrm{~g} / \mathrm{d}$ v. $2.3 \mathrm{~g} / \mathrm{d}$} & \multicolumn{2}{|c|}{$\begin{array}{c}\text { Active }(2.3 \text { and } 1.5 \mathrm{~g} / \mathrm{d}) \\
\text { v. placebo }\end{array}$} \\
\hline & Reduction & $95 \% \mathrm{Cl}$ & Reduction & $95 \% \mathrm{Cl}$ & Reduction & $95 \% \mathrm{Cl}$ & Reduction & $95 \% \mathrm{Cl}$ \\
\hline \multicolumn{9}{|c|}{ Absolute reduction $(\mathrm{mmol} / \mathrm{l})$} \\
\hline Total cholesterol & 0.25 & $0.07,0.43$ & 0.23 & $0.06,0.41$ & 0.02 & $-0.17,0.20$ & 0.24 & $0.09,0.40$ \\
\hline LDL-cholesterol & 0.17 & $-0.00,0.35$ & 0.15 & $-0.02,0.32$ & 0.02 & $-0.16,0.21$ & $0 \cdot 16$ & $0.01,0.32$ \\
\hline HDL-cholesterol & -0.01 & $-0.09,0.07$ & -0.01 & $-0.08,0.05$ & 0.01 & $-0.07,0.08$ & -0.01 & $-0.07,0.05$ \\
\hline TAG & 0.22 & $-0.19,0.62$ & 0.03 & $-0.51,0.58$ & 0.19 & $-0.28,0.65$ & 0.12 & $-0.29,0.54$ \\
\hline \multicolumn{9}{|c|}{ Proportional reduction (\%) } \\
\hline Total cholesterol & 4.4 & & $3 \cdot 8$ & & 0.5 & & $4 \cdot 1$ & \\
\hline Significance & $P=0.01$ & & $P=0.03$ & & $P=0.44$ & & $P<0.005$ & \\
\hline LDL-cholesterol & 3.4 & & 2.5 & & 0.8 & & 3.0 & \\
\hline Significance & $P=0.03$ & & $P=0.12$ & & $P=0.45$ & & $P=0.03$ & \\
\hline HDL-cholesterol & $-2 \cdot 7$ & & 0.0 & & $-2 \cdot 6$ & & $-1 \cdot 3$ & \\
\hline Significance & $P=0.66$ & & $P=0.47$ & & $P=0.37$ & & $P=0.87$ & \\
\hline TAG & 8.8 & & -2.8 & & 11.6 & & 2.9 & \\
\hline Significance & $P=0.45$ & & $P=0.39$ & & $P=0.87$ & & $P=0.35$ & \\
\hline
\end{tabular}

unlikely reason for the reduced effectiveness since the higher dose was at a level expected to produce maximal effects ${ }^{5}$.

Since the present study was a randomised controlled trial with good adherence and almost entirely complete follow up it is very unlikely that the results are importantly biased. Some confidence in this assumption is provided by the results themselves which, magnitude of effect on total and LDL-cholesterol aside, show consistent and expected directions of effect of plant sterols on all lipid parameters for both doses. In addition, the lipid assays done for the present study, which used direct measurement of all lipid components with an analytical system certified by the United States Center for Disease Control Cholesterol Reference Method Laboratory Network, are very unlikely to have introduced random or systematic error.

In conclusion, the present study has shown clear effects of plant sterol-enriched milk tea on blood lipid concentrations in a healthy urban Chinese population with above-average fat consumption and blood cholesterol concentrations. The effects on blood lipids were less than anticipated and the reasons for this are unclear but likely to be attributable to some aspect of either the novel population studied or the novel food format utilised. A study comparing the effects of the milk tea against another food format in Chinese individuals or a study of the milk tea food format in a Western population might provide significant additional insight. It is important to note, however, that while the changes in blood lipid concentrations were not as great as those anticipated these data do suggest that health gains would be achieved with the use of plant sterols in Chinese populations. Even moderate reductions in blood lipid concentrations would be anticipated to prevent serious cardiovascular events, particularly if treatment were targeted towards those at high risk of CVD or implemented population-wide.

\section{Acknowledgements}

Project funding was provided by Unilever Foods (China) Co. Ltd, B. C. N. was supported by a Career Development Award from the National Heart Foundation of Australia and N. Y. L. by a scholarship from the National Health and Medical
Research Council of Australia. The cardiovascular research activities of the George Institute are also supported by a programme grant awarded by the National Health and Medical Research Council of Australia.

B. C. N., N. Y. L., K. L., M. G. and H. O. F. M. designed the study. K. L. and Z. Q. managed the conduct of the project in China, with N. Y. L. and B. C. N. providing technical support from Australia. L. F. and N. Y. L. did the statistical analysis. The initial draft of the paper was prepared by N. Y. L. and B. C. N. with subsequent comments from all authors incorporated in the final draft. M. G., I. D. and H. O. F. M. are employed by the Unilever Food and Health Research Institute in the Netherlands. The remainder of the authors have no conflict. Furthermore, the contractual arrangement with Unilever clearly specified that there was no requirement for these authors to incorporate any comments or suggestions made by the Unilever employees.

Suzanne Ryan and Kathy Jane provided remote data management services from the George Institute for International Health in Sydney, Australia, Rong Han provided local project coordination in Beijing, China and Laurent Billot provided additional support for the statistical analysis.

The trial registration can be found at clinicaltrials.gov; identifier NCT00145717; http://www.clinicaltrials.gov/ct/show/ NCT00145717?order $=1$

\section{References}

1. Anonymous (1995) Cholesterol, diastolic blood pressure, and stroke: 13,000 strokes in 450,000 people in 45 prospective cohorts. Prospective studies collaboration. Lancet 346, $1647-1653$.

2. Li YH, Li Y, Davis CE, Chen Z, Tao S, Folsom AR, Bachorik P, Stamler J \& Abernathy JR (2002) Serum cholesterol changes from 1983-1984 to 1993-1994 in the People's Republic of China. Nutr Metab Cardiovasc Dis 12, 118-126.

3. Lichtenstein AH \& Deckelbaum RJ (2001) Stanol/sterol estercontaining foods and blood cholesterol levels. A statement for healthcare professionals from the Nutrition Committee of the Council on Nutrition, Physical Activity, and Metabolism of the American Heart Association. Circulation 103, 1177-1179. 
4. Trautwein EA, Duchateau GS, Lin Y, Mel'nikov SM, Molhuizen HO \& Ntanios FY (2003) Proposed mechanisms of cholesterol-lowering action of plant sterols. Eur J Lipid Sci Technol 105, 171-185.

5. Katan MB, Grundy SM, Jones P, Law M, Miettinen TA \& Paoletti R (2003) Efficacy and safety of plant stanols and sterols in the management of blood cholesterol levels. Mayo Clin Proc 78, 965-978.

6. Miettinen TA, Puska P, Gylling H, Vanhanen H \& Vartiainen E (1995) Reduction of serum cholesterol with sitostanol-ester margarine in a mildly hypercholesterolemic population. $N$ Engl $J$ Med 333, 1308-1312.

7. Hyun YJ, King OY, Kang JB, Lee JH, Jang Y, Liponkoski L \& Salo P (2005) Plant stanol esters in low-fat yogurt reduces total and low-density lipoprotein cholesterol and low density lipoprotein oxidation in normocholesterolemic and mildly hypercholesterolemic subjects. Nutr Res 25, 743-753.

8. Simons L (2002) Additive effect of plant sterol-ester margarine and cerivastatin in lowering low density lipoprotein cholesterol in primary hypercholesterolemia. Am J Cardiol 90, 737-740.

9. Anonymous (2004) The Nutrition and Health Status of the Chinese People. Material for the Press Conference of the State Council Information Office, October 2004. Beijing, China: State Council Information Office.

10. Zhang X, Patel A, Horibe $\mathrm{H}$, Wu Z, Barzi F, Rodgers A, MacMahon S \& Woodward M; Asia Pacific Cohort Studies Collaboration (2003) Cholesterol, coronary heart disease, and stroke in the Asia Pacific region. Int J Epidemiol 32, 563-572.

11. He J, Gu D, Reynolds K, et al., InterASIA Collaborative Group (2004) Serum total and lipoprotein cholesterol levels and awareness, treatment, and control of hypercholesterolemia in China. Circulation 110, 405-411.

12. Woodward M (2005) Epidemiology: Study Design and Data Analysis, 2nd ed. Boca Raton, London, New York, Washington, DC: Chapman \& Hall/CRC.
13. Tan CE, Loh LM \& Tai ES (2003) Do Singapore patients require lower doses of statins? The SGH Lipid Clinic experience. Singapore Med J 44, 635-638.

14. Chung N, Cho SY, Choi DH, et al. (2001) STATT: a titrateto-goal study of simvastatin in Asian patients with coronary heart disease. Simvastatin Treats Asians to Target. Clin Ther 23, 858-870.

15. Shin M-J, Rim S-J, Jang Y, Choi D, Kang S-M, Cho S-Y, Kim SS, Kim D, Song K \& Chung N (2003) The cholesterol-lowering effect of plant sterol-containing beverage in hypercholesterolemic subjects with low cholesterol intake. Nutr Res 23, 489-496.

16. Denke M (1995) Lack of efficacy of low-dose sitostanol therapy as an adjunct to a cholesterol-lowering diet in men with moderate hypercholesterolemia. Am J Clin Nutr 61, 392-396.

17. Hallikainen MA (1999) Effects of 2 low-fat stanol ester-containing margarines on serum cholesterol concentrations as part of a low-fat diet in hypercholesterolemic subjects. Am J Clin Nutr 69, 403-410.

18. Jones PJ, Ntanios FY, Raeini-Sarjaz M \& Vanstone CA (1999) Cholesterol-lowering efficacy of a sitostanol-containing phytosterol mixture with a prudent diet in hyperlipidemic men. Am J Clin Nutr 69, 1144-1150.

19. Noakes M, Clifton PM, Doornbos AME \& Trautwein EA (2004) Plant sterol ester-enriched milk and yoghurt effectively reduce serum cholesterol in modestly hypercholesterolemic subjects. Eur J Nutr 44, 214-222.

20. Devaraj S, Jialal I \& Vega-Lopez S (2004) Plant sterol-fortified orange juice effectively lowers cholesterol levels in mildly hypercholesterolemic healthy individuals. Arterioscler Thromb Vasc Biol 24, e25-e28.

21. Jones PJH, Vanstone CA, Raeini-Sarjaz M \& St-Onge M-P (2003) Phytosterols in low- and nonfat beverages as part of a controlled diet fail to lower plasma lipid levels. J Lipid Res 44, 1713-1719. 